\title{
MACHINE LEARNING ALGORITHM FOR SURFACE QUALITY ANALYSIS OF FRICTION STIR WELDED JOINT
}

\author{
AKSHANSH Mishra ${ }^{1 *}$ \\ ${ }^{1}$ Politecnico Di Milano, Department of Mechanical Engineering, Milan, Italy, e - mail: \\ akshansh.frictionwelding@gmail.com
}

\begin{abstract}
The Friction Stir Welding process usually produces weld members of good quality compared to composite weld made with a standard welding process. However, there is a possibility of the formation of various defects if the input parameters are not properly selected. In the recent case study, an image-based feature recognition system using the Fourier conversion method which is a computer visual recognition tool is developed. Five types of filters like Ideal Filter, Butterworth Filter, Low Filter, Gaussian Filter, and High Pass Filter. The results showed that the high pass filter has more ability to detect surface defects compared to the other four filters. It has also been observed that the Ideal filter has a lot of distortions compared to the Gaussian Filter and the Butterworth Filter.
\end{abstract}

KEYWORDS: Machine Learning; Machine Vision; Friction Stir Welding; Surface Defects

\section{Introduction}

Machine vision is a system engineering discipline that uses multiple cameras to automatically detect objects in the production area. The data extracted from the image analysis is also used to control the production process. An example would be, a camera used to capture any activity performed on a meeting line. In that case, the camera is designed to check the size, shape, or color of any object. In the worst case of equipment failure or malfunction, the work has stopped. Any material-based business should consider the care of machinery or equipment required. Adjustment is defined as the process of using IoT and machine learning to predict when assets need to be stored. This enables the manufacturer to reduce the downtime and use the equipment for life. 3D machine viewing systems usually consist of several cameras or laser sensing sensors. Multi-camera 3D view systems have multiple cameras installed in various locations and provide directional information to the robot. The big problem with visual data is that it is difficult to understand. For this reason, visual data is presented as a dark online story in the same way as a black story in physics [14].

Computer vision is a multidisciplinary branch that explores the various areas of science, engineering, and technology. The history of computer viewing dates back to about 543 million years ago. At that time the earth was very wet and few species of animals floated in the ocean. The animals did not move very well at that time they had no eyes. So whenever food is swimming nearby, they catch it or they just wander off. Something remarkable happened some 540 million years ago. From the study of fossils, zoologists found that in a very short time, the number of species became extinct. Andrew Parker, an Australian zoologist, studied the cause and suggested one of the most satisfying theories on fossil studies. He concluded that 540 million years ago the first animal evolved and that the beginning of the vision began a phase of explosive speculation. After 540 million years, the vision has grown into the largest sensory system in almost all animals, especially intelligent animals as humans. We have about 50 percent of the neurons in our cortex involved in the 
visual processing of the largest sensory system that enables us to survive, move, function, communicate, and manage objects [5-8]. Li et al. [9] have proposed image processing technology to detect surface defects in apples. Adel et al. [10] developed a computer screening program for detecting wood defects using a color analysis algorithm. Sarkar et al. [11] is designed to test a computer-based concept of tomato quality segmentation in new markets. The computer monitoring system has also been used in the Friction Stir Welding process [12]. Friction Stir Welding process is a solid state green technology used for joining the alloys which are difficult to join by conventional welding process [13]. The schematic representation of Friction Stir Welding process is shown in Figure 1. Mishra et al. [14] used the convolutional neural network to separate the texture of the Friction Stir Welded joint from the common welded joint.

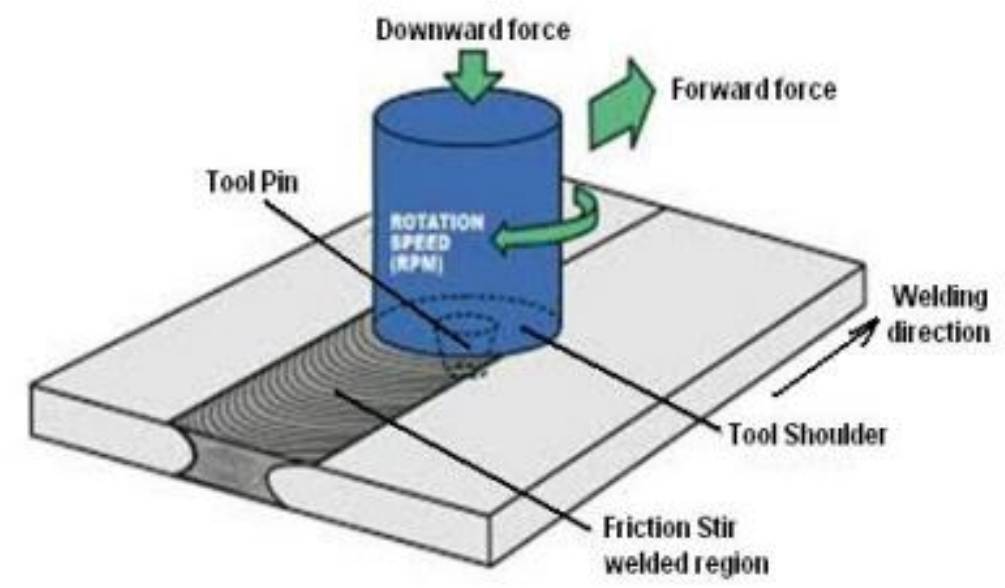

Fig. 1 Schematic representation of Friction Stir Welding process [15]

In the recent work, Fourier transformation which is a Computer vision tool utilized by using Python programming executed on Google Colaboratory platform for the edge detection of Friction Stir Welded joints.

\section{Material and Methods}

The structure and properties such as local changes in the image i.e. Edges are the main regions of interest in the present work. Edge formation generally occurs at the boundary between two different regions in an image due to a local change in the image intensity which occurs due to discontinuity in the first derivative of the image intensity. So, the first step to extract the necessary features from the given image is the Edge detection technique. A step edge is linked with a local peak value in the first derivative in a one-dimensional view. An array of samples of some continuous function of an image intensity constitute an image and gradient can measure a change in these functions. By using a discrete approximation to the gradient, any slight change in the gray values of an image can be easily detected. The twodimensional equivalent of the first derivative is represented by a gradient as shown in Equation 1.

$$
\mathrm{G}[\mathrm{f}(\mathrm{x}, \mathrm{y})]=\left[\begin{array}{l}
G_{x} \\
G_{y}
\end{array}\right]=\left[\begin{array}{l}
\frac{\partial f}{\partial x} \\
\frac{\partial f}{\partial y}
\end{array}\right]
$$


In the Equation 1, $\mathrm{G}[\mathrm{f}(\mathrm{x}, \mathrm{y})]$ is a vector which points in the direction of the maximum rate of the increase of the function of $f(x, y)$ and its magnitude and direction can be expressed by Equation 2 and 3.

$$
\begin{gathered}
\mathrm{G}[\mathrm{f}(\mathrm{x}, \mathrm{y})]=\sqrt{G_{x}^{2}+G_{y}^{2}} \\
\alpha(\mathrm{x}, \mathrm{y})=\tan ^{-1}\left(\begin{array}{c}
G_{y} \\
G_{x}
\end{array}\right)
\end{gathered}
$$

In the research work, AA 6060-T5 aluminum alloys were similar welded by the Friction Stir Welding Process. The dimensions of the base alloy plates joined were $150 \mathrm{~mm} \mathrm{X} 100 \mathrm{~mm}$ X $6 \mathrm{~mm}$. H13 tool material was preferred because it's harder than the base metal alloy to be joined. Tool rotational speed (RPM), welding speed $(\mathrm{mm} / \mathrm{min})$ and the axial force $(\mathrm{KN})$ are the key input parameters for obtaining the sound quality of Friction Stir Welded joint. For joining of the similar base metal alloy plates tool rotational speed of $2000 \mathrm{rpm}$, welding speed of $200 \mathrm{~mm} / \mathrm{min}$ and an axial force of $1.5 \mathrm{KN}$ were selected. The digital high quality image of the welded joint was taken in RGB format and it was further cropped to a particular size as shown in Figure 2.

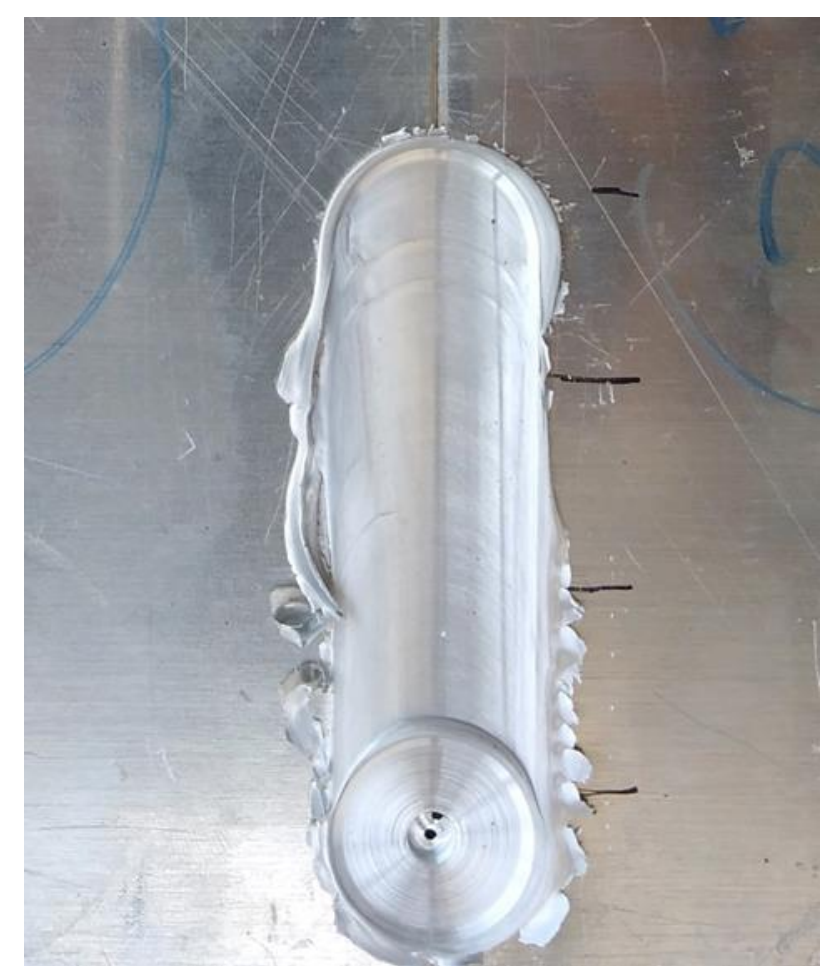

Fig 2. Cropped image of Friction Stir Welded joint

If a function $\mathrm{f}(\mathrm{x})$ is defined on $[\mathrm{c}, \mathrm{c}+21]$ or $(\mathrm{c}, \mathrm{c}+21)$ of length ' 21 ', is a periodic function with arbitrary period ' $2 \mathrm{l}$ ' then the fourier series of $\mathrm{f}(\mathrm{x})$ is

$$
\mathrm{f}(\mathrm{x})=\frac{a_{0}}{2}+\sum_{n=1}^{\infty} a_{n} \cos \left(\frac{n \pi x}{l}\right)+\sum_{n=1}^{\infty} b_{n} \sin \left(\frac{n \pi x}{l}\right)
$$

where,

$$
\begin{aligned}
a_{0} & =\frac{1}{l} \int_{c}^{c+2 l} f(x) \cdot d x \\
a_{n} & =\frac{1}{l} \int_{c}^{c+2} f(x) \cos \left(\frac{n \pi x}{l}\right) \cdot d x \\
b_{n} & =\frac{1}{l} \int_{c}^{c+2 l} f(x) \sin \left(\frac{n \pi x}{l}\right) \cdot d x
\end{aligned}
$$


The process flow chart for subjecting the image to fourier transformation is shown in the Figure 3.

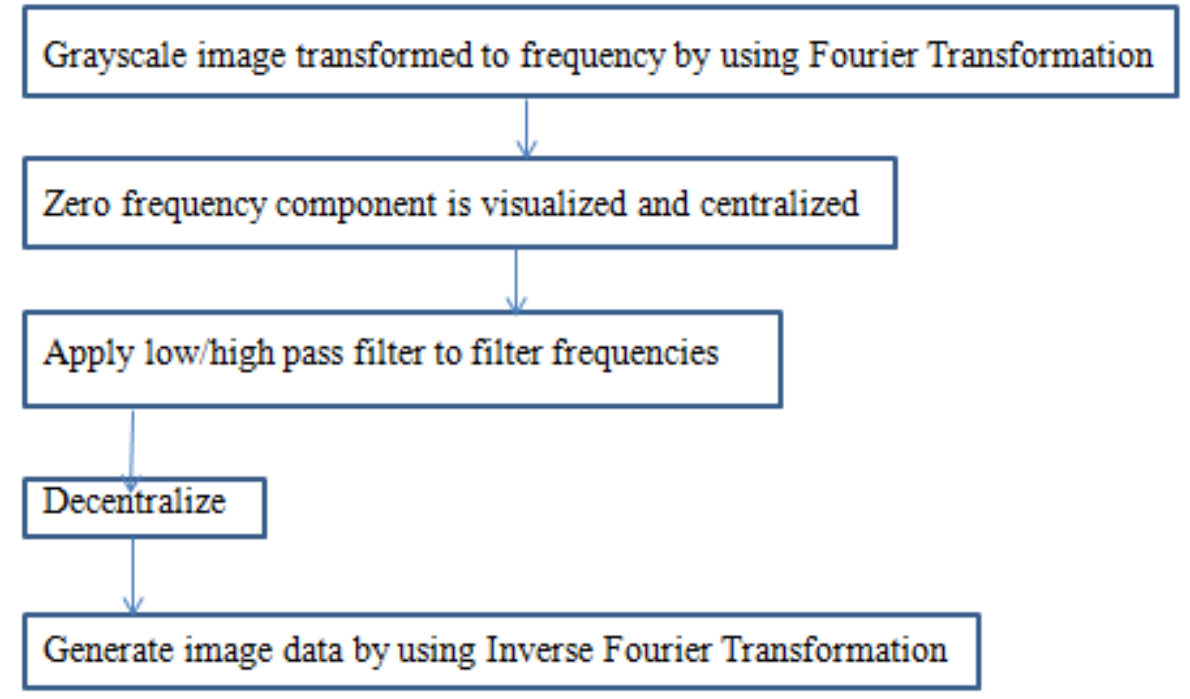

Fig 3. Steps to obtain Fourier transformed images

\section{Results and Discussions}

Image pixels are discontinuous in nature, so digital images are considered as a discrete. In the research study, Fast Fourier Transformation (FFT) is used instead of Discrete Fourier Transformation (DFT) because the latter is too slow for the image processing task. Figure 4 shows the subjection of the Fourier Transformation to the given friction stir welded joint image.
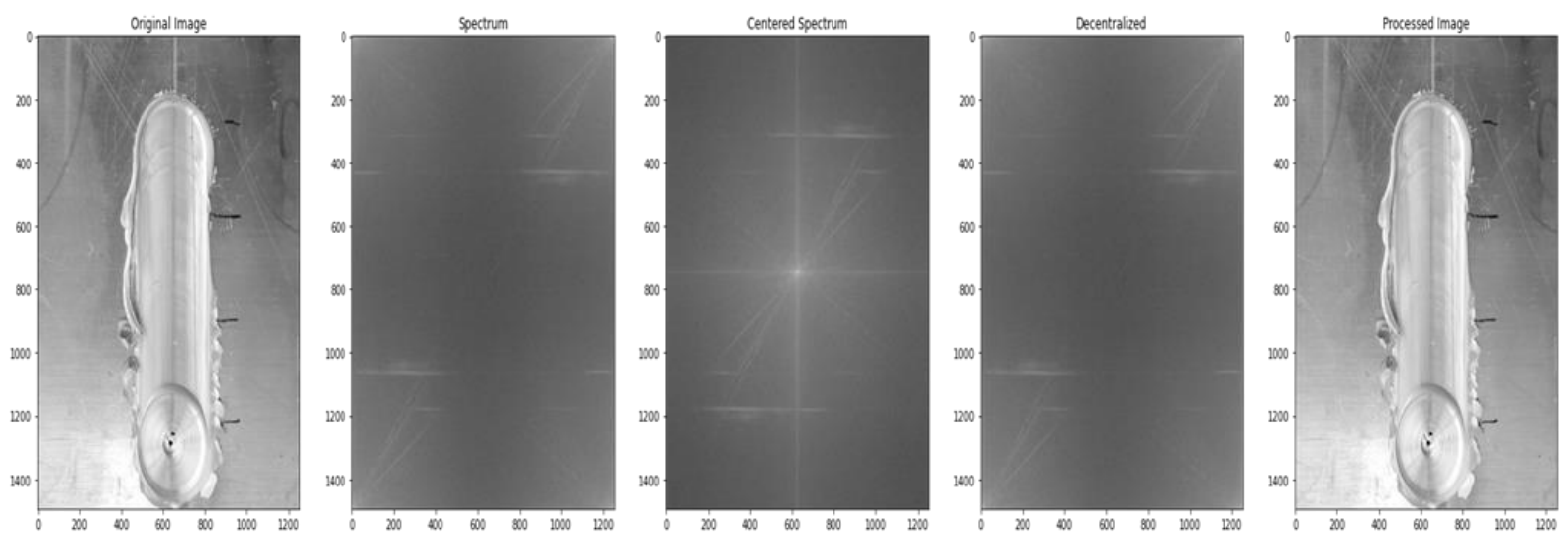

Fig.4. Image subjected to Fourier Transformation. a) Original Image.

b) Fast Fourier Transform visual output. c) Centralized.

d) Decentralized. e) Inverse Fast Fourier Transformation

The output obtained from Fast Fourier Transformation is very difficult to visualize because they consist of complex numbers array. So the result is transformed into two dimensional spaces. Spectrum and phase angle are two methods to visualize the result of Fast Fourier Transformation as shown in the Figure 5. From the Figure 5 a) it is observed that on the four corners there are some symmetric patterns which can be translated to the center of the image in the upcoming step. Low frequencies are represented in the corner of spectrum image while high power frequency is represented in the white area of the spectrum image. 

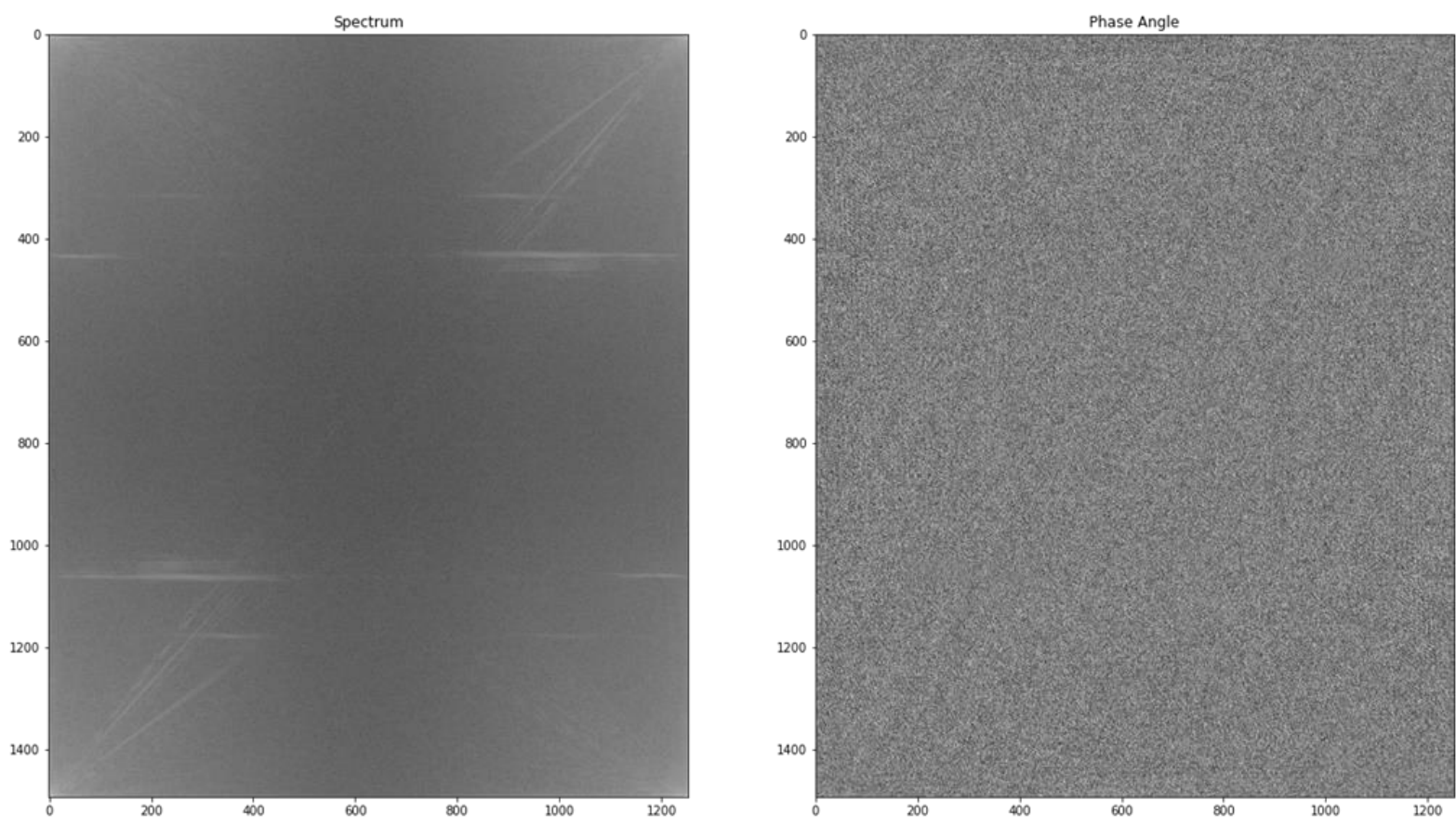

Fig.5. a) Spectrum b) Phase angle

From Figure $5 \mathrm{~b}$ ) it is difficult to extract any distinguishing patterns. Shape characteristics are an essential feature of the image which is preserved by the phase. In order to implement various filters, the zero frequency component was shifted to the center of spectrum. Figure 6 represents the implementation of the low pass filter to the obtained spectrum.
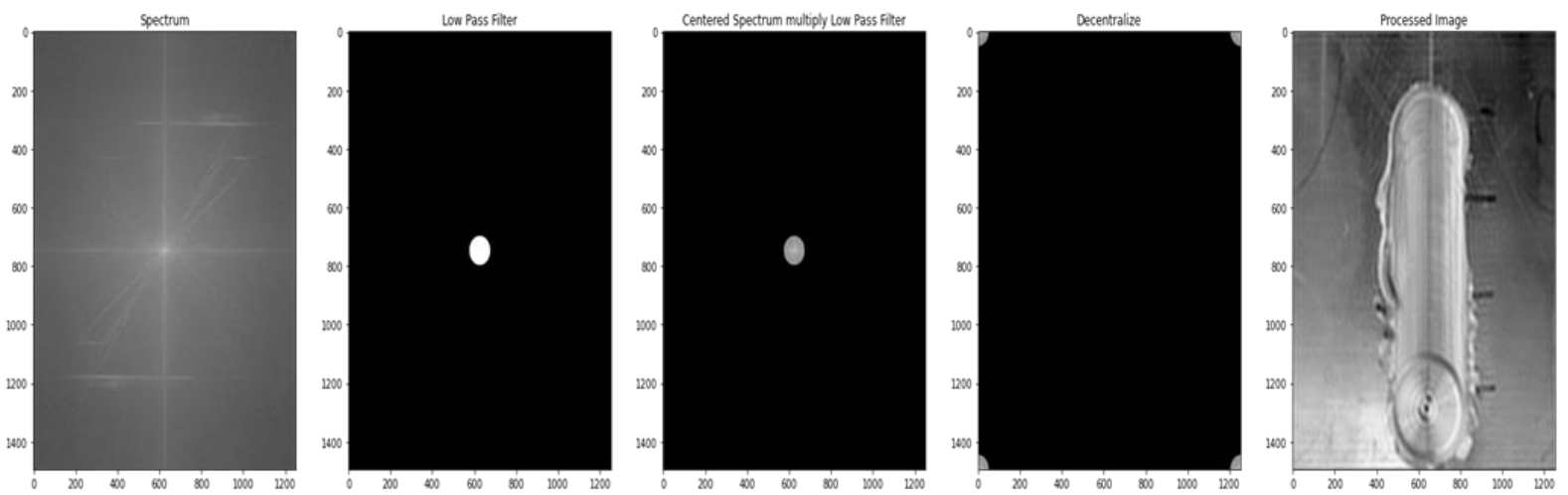

Fig.6. Low pass filter applied to the spectrum

Low frequencies are allowed to pass through the low pass filters. Low pass filter is useful for removing the noises in the images as noises are high frequencies contents. Figure 7 represents the implementation of the high pass filter to the obtained spectrum.

High frequencies are passed though high pass filter. Pixels values which change drastically represent high frequencies. Edges in the given image are captured by using the high pass filters as shown in the Figure 7 e). High pass filters can easily recognize any change in a given image. Equation 5 represents the formula of ideal low pass filter.

$$
H(u, v)= \begin{cases}1 & \text { if } D(u, v) \leq D_{0} \\ 0 & \text { if } D(u, v)>D_{0}\end{cases}
$$

In the above equation, $D_{0}$ is a some positive constant and $\mathrm{D}(\mathrm{u}, \mathrm{v})$ is the distance between the center of the frequency triangle and a point $(\mathrm{u}, \mathrm{v})$ in a frequency domain as shown in Figure 8. 

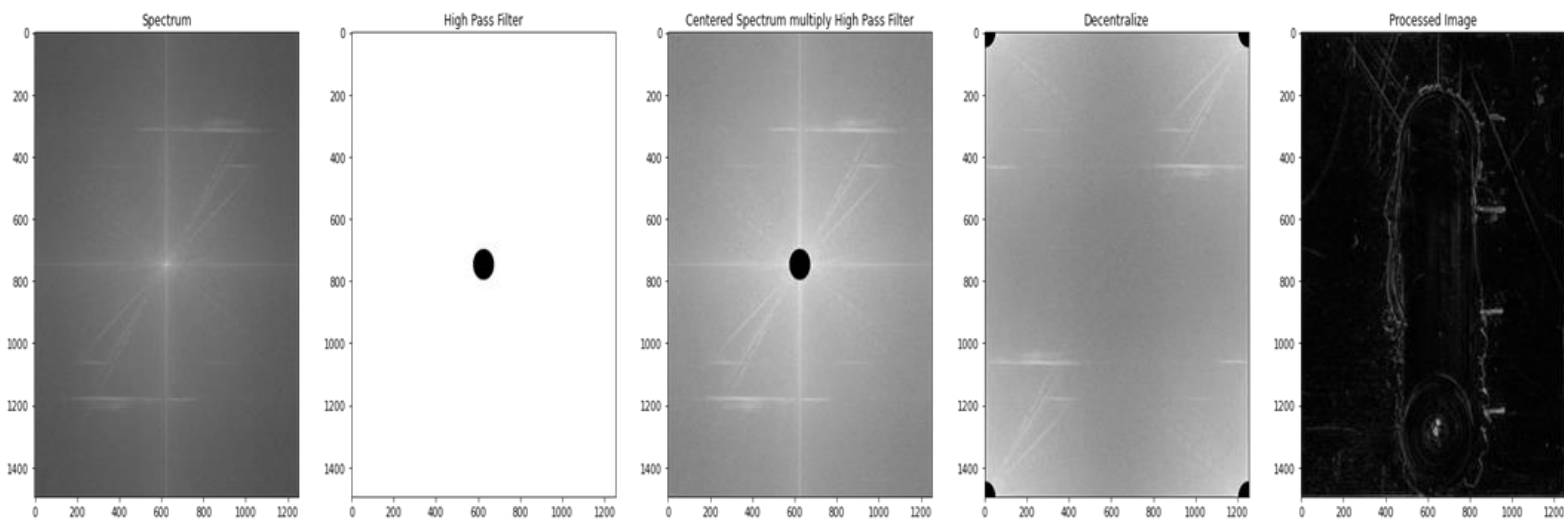

Fig.7. Spectrum subjected to High Pass Filter
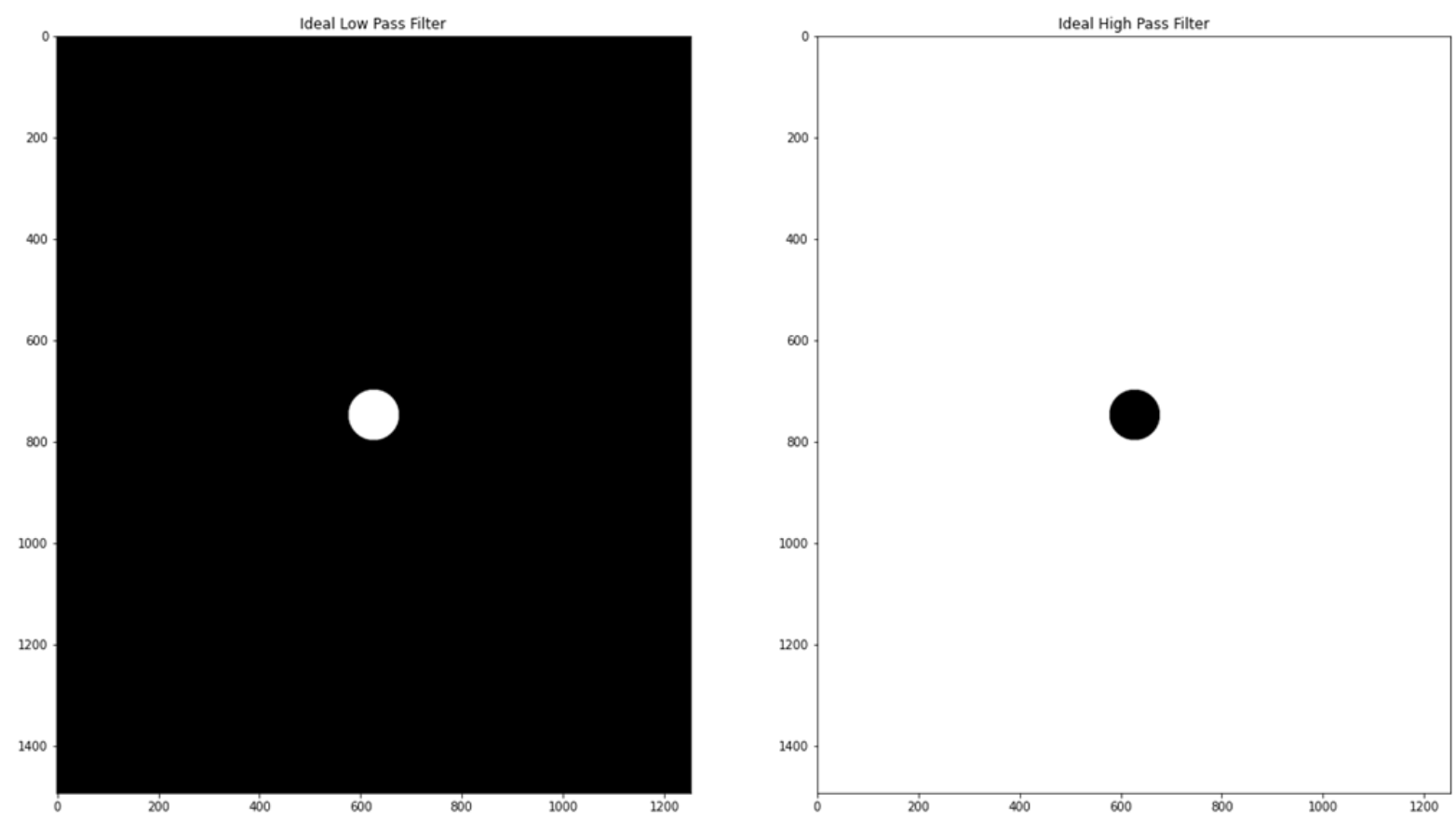

Fig.8. a) Low pass filter with positive constant $=50$.

b) High pass filter with positive constant $=50$

Equation 6 represents the formula for ideal high pass filter.

$$
H(u, v)= \begin{cases}0 & \text { if } D(u, v) \leq D_{0} \\ 1 & \text { if } D(u, v)>D_{0}\end{cases}
$$

Clear cut off can be achieved between filtered and passed frequencies by using Butterworth filter which is totally different from ideal pass filter. Equation 7 and Equation 8 represents the formula for Butterworth low pass and Butterworth high pass filter respectively. It is also observed in the both equations that a new parameter ' $n$ ' is introduced in the given function whose manipulation affects the clearness of the cut off between the filtered and the passed frequencies as shown in the Figure 9 and 10.

$$
H(u, v)=\frac{1}{1+\left[D(u, v) / D_{0}\right]^{2 n}}
$$




$$
H(u, v)=\frac{1}{1+\left[D_{0} / D(u, v)\right]^{2 n}}
$$
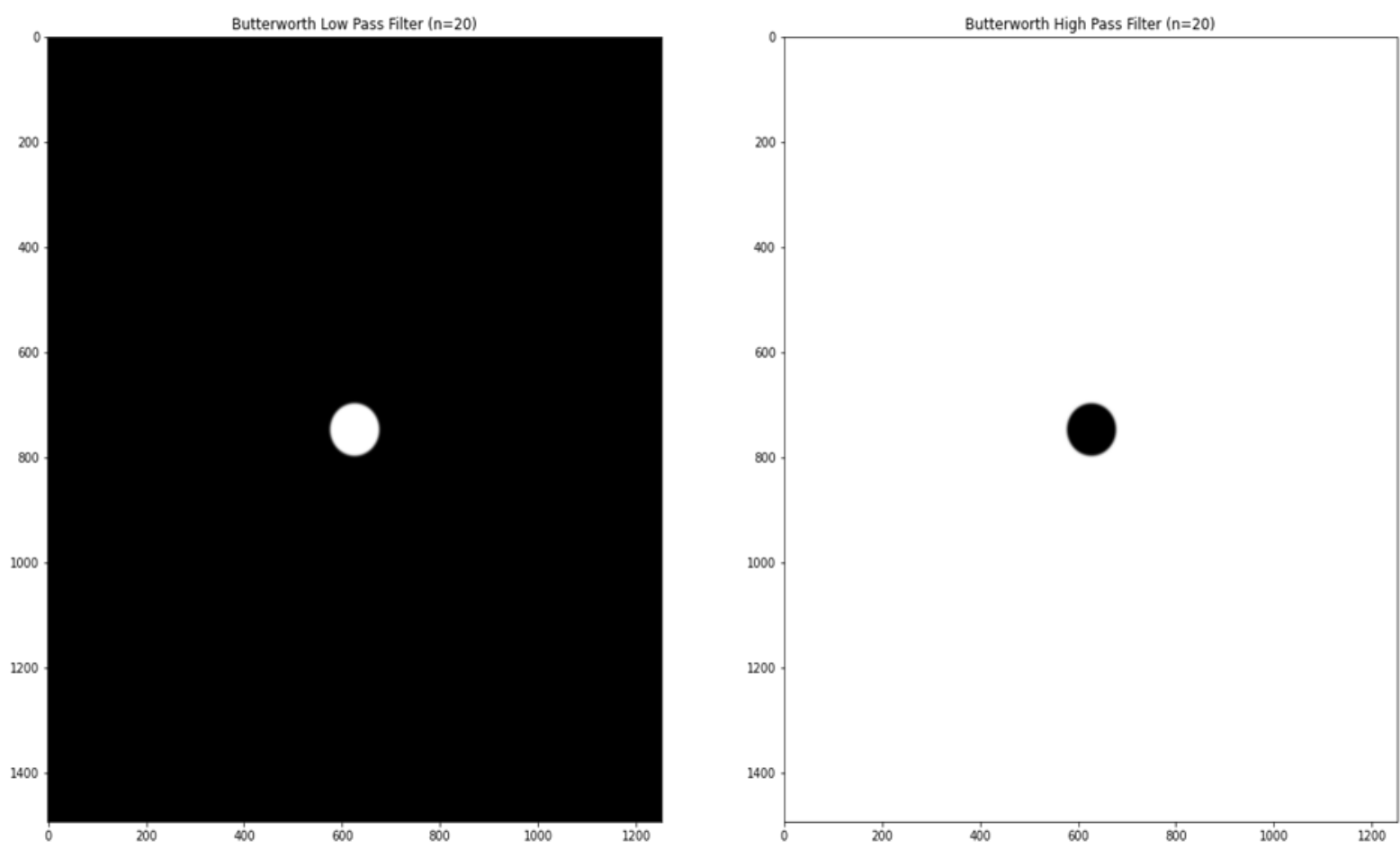

Fig.9. a) Butterworth low pass filter with positive constant $=50$ and $n=20$.

b) Butterworth high pass filter with positive constant $=50$ and $n=20$
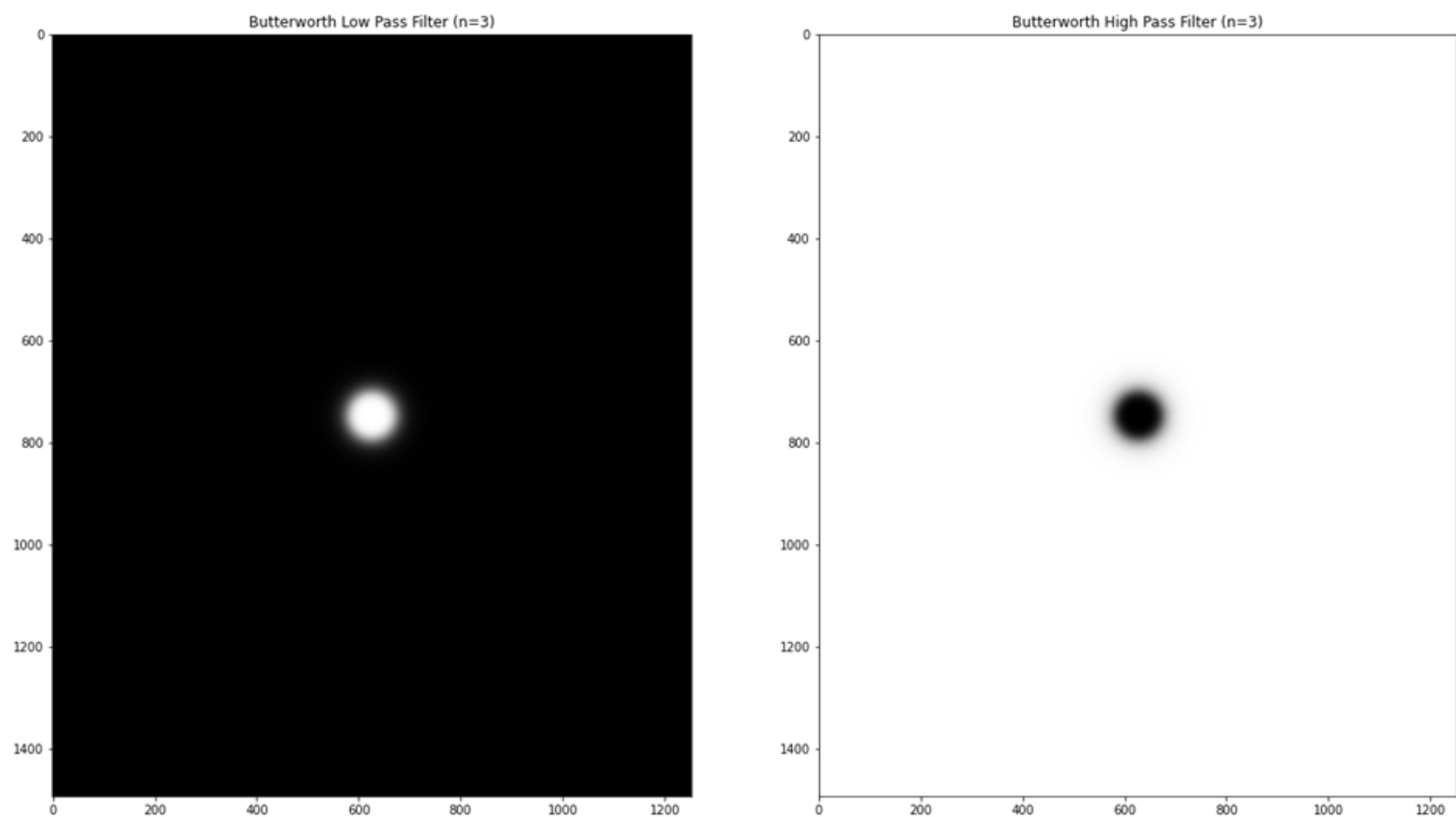

Fig.10. a) Butterworth low pass filter with $n=3$. b) Butterworth high pass filter with $n=3$

On the other hand, in comparison to the Butterworth filter, Gaussian filter provides smoother cut off. Equation 9 and Equation 10 represents the formulas for Gaussian Low pass and Gaussian High pass filter respectively. 


$$
\begin{aligned}
& H(u, v)=e^{-D^{2}(u, v) / 2 D_{0}^{2}} \\
& H(u, v)=1-e^{-D^{2}(u, v) / 2 D_{0}^{2}}
\end{aligned}
$$

Gaussian filter results smoother processed images because the cut off between filtered and passed frequencies are blurry in nature which is shown in the Figure 11.
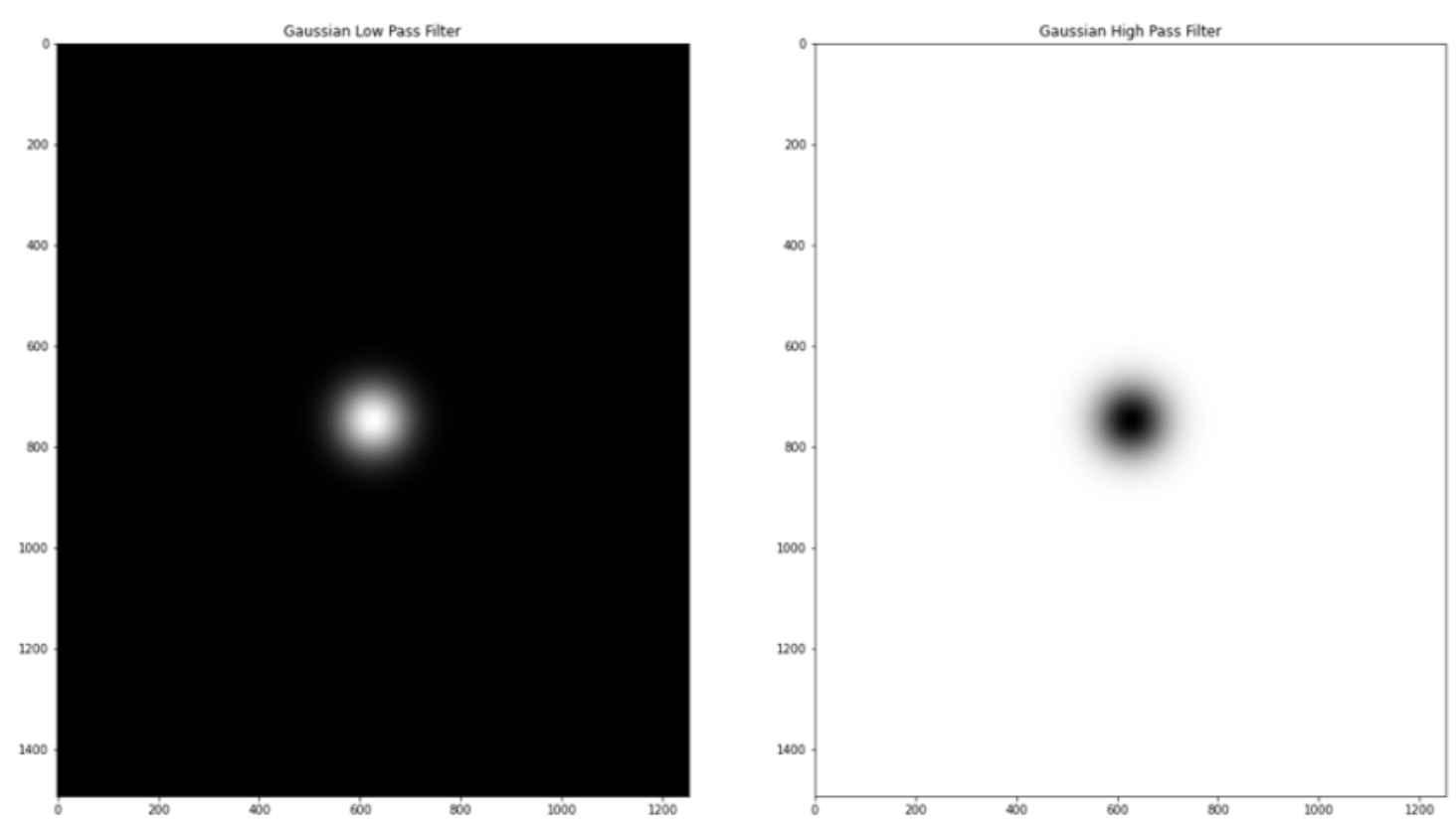

Fig.11. a) Gaussian low pass filter with positive constant=50.

b) Gaussian high pass filter with positive constant $=50$

Figure 12 shows that in comparison to the other filters, High pass filter results better edge detection accuracy in the friction stir welded joint image.
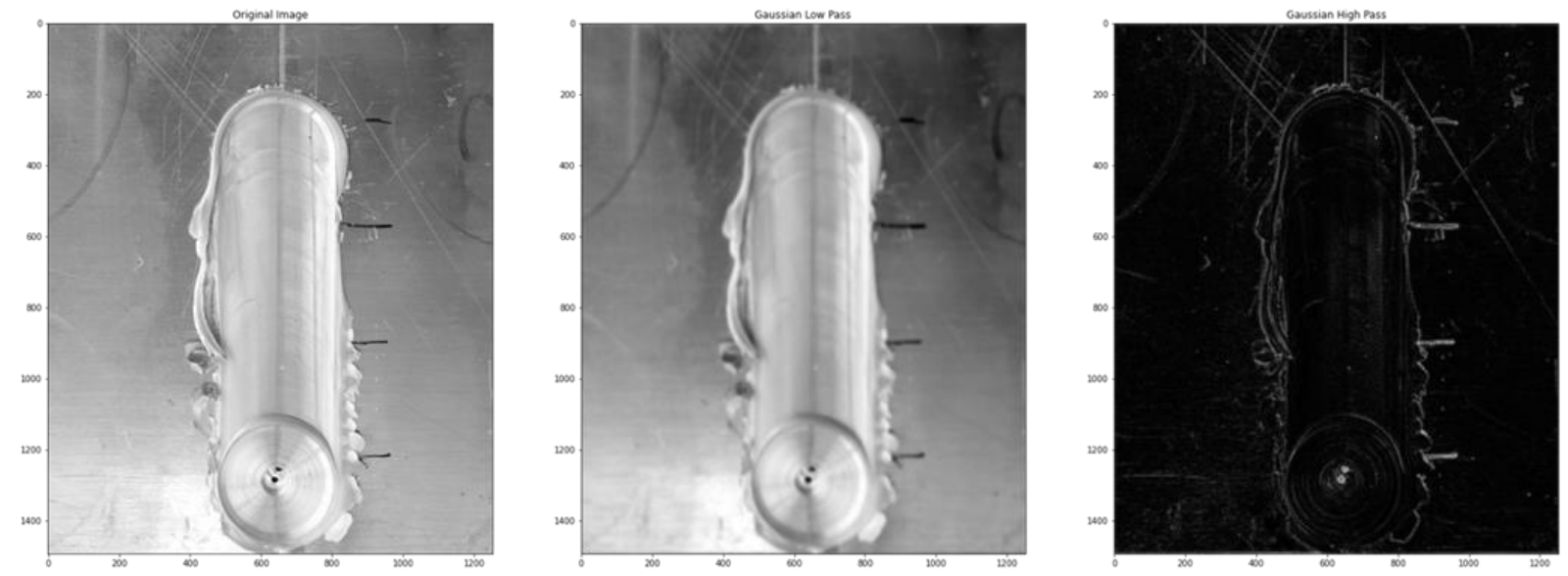

Fig.12. Edge detection by using High pass filter

\section{CONCLUSION}

The research study proposes a novel approach for surface edge detection in the Friction Stir Welded joint by using computer vision technique. It is observed that the Gaussian filter performs better than the Ideal filter and Butterworth filter because it results less distortion and 
more smoothness to the processed image. Also it is observed that the high pass filter has better advantage over other filters in terms of detecting surface edges. In the future research, Fourier transformation can play a vital role for image processing task by providing more flexibility.

\section{REFERENCES}

[1] Szegedy, C., Vanhoucke, V., Ioffe, S., Shlens, J. and Wojna, Z. "Rethinking the inception architecture for computer vision", In Proceedings of the IEEE conference on computer vision and pattern recognition, pp. 2818 - 2826, 2016.

[2] Parker, J. R. "Algorithms for image processing and computer vision", John Wiley \& Sons, 2010.

[3] Granlund, G. H. and Knutsson, H. "Signal processing for computer vision", Springer Science \& Business Media, 2013.

[4] Forsyth, D. A., Ponce, J. "Computer vision: a modern approach", Prentice Hall Professional Technical Reference, 2002.

[5] Kruger, N., Janssen, P., Kalkan, S., Lappe, M., Leonardis, A., Piater, J., RodriguezSanchez, A. J., Wiskott, L. "Deep hierarchies in the primate visual cortex: What can we learn for computer vision?", IEEE transactions on pattern analysis and machine intelligence 35 (8), pp. 1847 - 1871, 2012.

[6] Weinland, D., Ronfard, R., Boyer, E. "Free viewpoint action recognition using motion history volumes", Computer vision and image understanding 104 (2 - 3), pp. 249 - 257, 2006.

[7] Ahad, M. A. R., Tan, J. K., Kim, H., Ishikawa, S. "Motion history image: its variants and applications", Machine Vision and Applications 23 (2), pp. 255 - 281, 2012.

[8] Huang, T. "Computer vision: Evolution and promise", 1996.

[9] Li, Q., Wang, M. and Gu, W. "Computer vision based system for apple surface defect detection", Computers and electronics in agriculture 36 (2-3), pp. 215 - 223, 2002.

[10] Adel, M., Wolf, D., Vogrig, R., Husson, R. "Evaluation of colour spaces in computer vision application of wood defects detection", In Proceedings of IEEE Systems Man and Cybernetics Conference-SMC 2, pp. 499 - 504, 1993.

[11] Sarkar, N., Wolfe, R. R. "Computer vision based system for quality separation of fresh market tomatoes", Transactions of the ASAE 28 (5), pp. 1714 - 1718, 1985.

[12] Murthy, V., Ullegaddi, K., Mahesh, B., Rajaprakash, B. M. "Application of image processing and acoustic emission technique in monitoring of friction stir welding process”, Materials Today: Proceedings 4 (8), pp. 9186 - 9195, 2017.

[13] Srinivasa Rao, M., Ramanaiah, N. "Optimization of Process Parameters for FSW of AlMg-Mn-Sc-Zr Alloy Using CCD And RSM", Strojnícky časopis - Journal of Mechanical Engineering 68 (3), pp. 195 - 224, 2018. DOI: 10.2478/scjme-2018-0035

[14] Mishra, A., Nagpal, K. "Convolutional Neural Network for Image Processing of Friction Stir Welded and Conventional Welded Joints Texture", Int. J. Hum. Comp. Inter. Data Min 2 (1\&2), pp. 5 - 9, 2019.

[15] Vishwanath, M. M., Lakshamanaswamy, N., Ramesh, G. K. "Numerical Simulation of Heat Transfer Behavior of Dissimilar AA5052-AA6061 Plates in Fiction Stir Welding: 
An Experimental Validation", Strojnícky časopis - Journal of Mechanical Engineering 69 (1), pp. 131 - 142, 2019. DOI: 10.2478/scjme-2019-0011 